\title{
Dysregulation of Interleukin 23 Receptor Expression in Kidney Allografts Associated with Composite Outcome
}

Kuang-Chin Hsiao ${ }^{1,2}$, Wan-Ru Chao ${ }^{2,3}$, Jen-Pi Tsai ${ }^{4}$, Mei-Chin Wen ${ }^{5}$, Jong-Da Lian ${ }^{6}$, Wen-Chin Lee ${ }^{1}$, Jong-Yu Huang ${ }^{1}$, Shun-Chi Chang ${ }^{1}$ and Horng-Rong Chang ${ }^{2,6}$

${ }^{1}$ Division of Nephrology, Department of Medicine, Show Chwan Memorial Hospital, Changhua, Taiwan

${ }^{2}$ Institute of Medicine, Chung Shan Medical University, Taichung, Taiwan

${ }^{3}$ Department of Pathology, Chung Shan Medical University, Taichung, Taiwan

${ }^{4}$ Department of Nephrology, Buddhist Dalin Tzu Chi General Hospital, Chiayi, Taiwan

${ }^{5}$ Department of Pathology, Taichung Veterans General Hospital, Taichung, Taiwan

${ }^{6}$ Division of Nephrology, Department of Medicine, Chung Shan Medical University Hospital, Taichung, Taiwan

*Corresponding author: Horng-Rong Chang, Division of Nephrology, Department of Medicine, Chung Shan Medical University Hospital, Address: No. 110, Sec. 1, Jianguo N. Road South District, Taichung 40201, Taiwan, Tel: +886-4-24739595 extn. 32620; Fax: +886-4-24739220 extn. 32624 ; E-mail: chrcsmu@gmail.com

Rec date: Sep 23, 2014, Acc date: Nov 17, 2014, Pub date: Nov 19, 2014

Copyright: (c) 2014 Hsiao KC, et al. This is an open-access article distributed under the terms of the Creative Commons Attribution License, which permits unrestricted use, distribution, and reproduction in any medium, provided the original author and source are credited.

\begin{abstract}
Background: Interleukin 23 (IL-23) and interleukin 23 receptor (IL-23R) play a role in the pathogenesis of multiple autoimmune processes and renal inflammation, but research has yet to clarify the histological association of IL-23/IL-23R and transplant kidney allografts.

Methods: Between July 2009 and August 2011, 31 renal transplant recipients who received sonography-guided kidney allograft biopsy were enrolled in this retrospective study. The patients were divided into two groups including group A (patients reaching composite outcome) and group B (patients not reaching composite outcome). The composite outcome was defined as serum creatinine (Scr) doubling and lower estimated glomerular filtration rate (eGFR). Specimens of 31 patients were examined by the immunohistochemical stain of IL-23 and IL-23R in allograft kidneys, and clinico-pathological associations were evaluated.

Results: Of the 31 patients, group A had 15 patients (48.3\%) and group B had 16 patients (52.7\%). Group A had significantly higher $\mathrm{SCr}$, lower eGFR, and low serum albumin $(p=0.024)$. Univariate analysis showed that group $A$ was negatively associated with atrophic glomerular mesangial cell cytoplasmic IL-23R expression $(p=0.044)$. The decreased expression of IL-23R could be due to higher acute antibody-mediated rejection with heavy proteinuria in our study. In other words, the more the glomerular damage due to antibody-mediated rejection, the less the expression of IL-23R in atrophic glomerular mesangial cell cytoplasma.
\end{abstract}

Conclusions: The patients with composite outcome may have decreased expression of IL-23R in atrophic glomerular mesangial cell cytoplasm.

Keywords: IL-23; IL-23 receptor; Immunohistochemistry stain; Renal transplantation

\section{Introduction}

A kidney transplant is the best choice for the treatment of end stage kidney disease. Rejection of transplanted tissues involves the interplay between mechanisms that maintain tolerance to the graft and factors that promote rejection. Acute rejection continues to be one of the most important causes of graft loss and involves the cellular and/or humoral immune response [1]. Cellular rejection is characterized morphologically by the presence of mononuclear cells in the interstitial, tubular, and glomerular compartments [1-3]. Moreover, humoral rejection is associated with vascular involvement (vasculitis), deposition of immunoglobulins (C4d deposition in peritubular capillaries), and activation of the complement cascade [4-6].

Patients with acute allograft rejection present with an acute rise in the Scr and lower eGFR. A rising Scr level, however, is a relatively late development in the course of a rejection episode and usually indicates the presence of significant histological damage [1]. New or worsening proteinuria may also be present with/without decreased serum albumin level, especially in the case of acute humoral rejection [6].

T lymphocytes not only play an essential role in the initiation of the cascade of mechanisms underlying rejection but also participate in mechanisms that maintain graft tolerance $[7,8]$. Naive CD4+ helper T cells have been shown to develop into at least 4 types of committed helper T cells, namely, T helper (Th) 1, Th2, Th17, and regulatory $\mathrm{T}$ cells $[3,5,9]$. Interleukin 23 (IL-23) affects interferon- $\gamma$ production (IFN- $\gamma$ ) by $\mathrm{T}$ and natural killer cells, activates memory $\mathrm{T}$ cells, stimulates Th1 cell responses, and enhances inflammation by stimulating the production of proinflammatory cytokines $[10,11]$.

IL-23 mediates these effects through binding a receptor composed of IL-12Rb1 and interleukin 23 receptor (IL-23R), with the latter being located on chromosome 1p31.3. IL-23R, which is the initial sensor of the IL-23 signal, also determines Th17cell expansion and in turn serves 
as an important gate for Th17 cell mediated autoimmune responses [12]. An accumulating body of literature reports that the presence of IL-23R gene polymorphism is associated with various autoimmune diseases such as rheumatoic arthritis, Crohn's disease, Grave's ophthalmopathy and graft-versus-host [13-16]. Recently, Tsai et al. [17] revealed an association between interleukin 23 receptor polymorphism and kidney transplant outcomes.

Based on the aforementioned background research, we hypothesize that IL-23 and IL-23R are related to the immuno-modulatory functions of transplanted kidneys. The aim of this study is thus to examine the clinico-pathological correlation of expressions of IL-23/ IL-23R in kidney transplant allografts.

\section{Materials and Methods}

\section{Study design and patients}

From July 2009 to August 2011, pathological specimens from 31 renal transplantation recipients who received sonography-guided kidney allograft biopsy were retrospectively recruited. Institutional review board approval of Chung Shan Medical University Hospital was obtained for the review of patients' medical records, data analysis and pathological specimens staining, and the need for informed consent was waived.

Patient age, gender, body mass index, status of cigarette smoking, hypertension, diabetic mellitus, hepatitis B, hepatitis C, blood pressure, dialysis mode before transplantation, immusupressant drug (tacrolimus and cyclosporin) and drug trough level were recorded. Labotory data including SCr, eGFR, hemoglobin, hemoglobin A1c (HBA1C), lipid titer, albumin, uric acid and dipstick urine protein. eGFR was calculated by the abbreviated Modification of Diet in Renal Disease formula (aMDRD): eGFR=186 x (serum Creatinine)-1:154 x (age)-0:203 x (0.742 if female). Chronic kidney disease was defined by K/DOQI guidelines [18].

\section{Primary composite outcome}

The primary outcome measured in this study was a composite endpoint of time to first event with a doubling of SCr or declining of eGFR of more than $30 \%$. The composite group (group A) was defined as reaching the doubling value of baseline SCr or declining of baseline eGFR of more than $30 \%$. The group B was defined as not reach composite outcome. A baseline SCr/eGFR was defined as the best stable level of SCr/eGFR within six months between allograft biopsy, and terminal SCr/eGFR was defined as the following of SCr/eGFR to September 15, 2013.

Doubling of SCr was defined as the first date to have the doubling value of baseline $\mathrm{SCr}$, and the declining of eGFR was defined as the first date when eGFR declined 30\% from baseline eGFR. Doubling of SCr was chosen as the primary composite outcome because it is a welldefined, clinically relevant outcome $[19,20]$. As recently recommended by the US Food and Drug Administration (FDA), we used the $30 \%$ decline in eGFR as an endpoint for kidney failure [21].

\section{Clinical pathological diagnosis}

The clinical pathological diagnosis was acute tubular injury $(\mathrm{n}=5)$, mild acute cellular rejection $(n=8)$, severe acute cellular rejection $(n=13)$ and antibody mediated rejection (ABMR) $(n=5)$. The severe acute cellular rejection was defined as Banff type acute cellular rejection IA, IB and IIA [22]. If there were more than two pathological diagnoses of ex calcineurin inhibitor nephrotoxicity, chronic fibrosis change, de novo glomerulonephritis, dibetic nephropathy or polyomavirus nephropathy in one renal specimen, we chose the major pathological result as the pathological diagnosis in this study.

\section{Tissue processing}

Pathologic material was processed by conventional histological procedures. The specimens were collected by sonography-guided kidney allograft biopsy. Each section was at least $2 \times 0.5 \mathrm{~cm} 2$. The formalin-fixed, paraffin-embedded tissues were cut into 4-mm hematoxylin- and eosin-stained ( $\mathrm{H} \& \mathrm{E}$ staining) sections and examined to evaluate the glomerular, renal tubular, and interstitial conditions. The scoring of fibrosis was based on Banff scoring for chronic lesions [22] with interstitial fibrosis score and glomular fibrosis score.

Each patient who recieved kidney allograft biopsy was received medical therapy according to the pathological diagnosis. When the renal insuffiency recovered, the patient was discharged with an outpatient department follow up. The remaining biopsy tissue was stained by innumohistochemical (IHC) of IL-23 and IL-23R. The study protocol was approved by the local Ethics Committee.

\section{Immunohistochemical staining}

Paraffin embedded kidney tissue sections (4-mm) on poly-1-lysinecoated slides were deparaffinized. After treatment with $3 \% \mathrm{H} 2 \mathrm{O} 2$ in methanol, the sections were hydrated with gradient alcohol and PBS, incubated in $10 \mathrm{mM}$ citrate buffer, and finally heated at $100 \mathrm{uC}$ for 20 min in PBS. Slides were incubated with the anti-IL-23 and IL-23R antibody (Santa Cruz, CA, USA) for $20 \mathrm{~min}$ at room temperature, and then with a horseradish peroxidase (HRP)/Fab polymer conjugate for another $30 \mathrm{~min}$. Then, slides were thoroughly washed three times with PBS, and the sites of peroxidase activity were visualized using 3, 3diamino-benzidine tetrahydrochloride as a substrate and hematoxylin as the counter stain.

\section{Semi-quantitative grading}

All IHC stain data were independently scored by two blinded pathologists using the following scale: $0=$ no staining, $1=$ mild staining, $2=$ moderate staining, $3=$ high staining (Figure 1). Every slide was examined entirely for nuclear and cytoplasmic IL-23/IL-23R stains in the normal and atrophic renal tubules, in the normal and atrophic glomeruli, and in the renal interstitium. Each $2 \times 0.5 \mathrm{~cm} 2$ section contained at least 10 glomerular areas, and the actual number of examined glomeruli was based on the sectioned tissue size. The results of nuclear and cytoplasmic staining were recorded separately. The intensity of IL-23 and IL-23R staining was classified as with staining $(1,2$ and 3$)$ or without staining $(0)$.

\section{Statistical analysis}

Continuous and categorical data were expressed as median (25\%-75\%) and as proportions, respectively. Categorical variables were analyzed by the chi-square test or Fisher's exact test. Spearman's rank correlation coefficient was used for association between clinical variables when reaching composite outcome. A p-value less than 0.05 was considered statistically significant. All data were analyzed using SPSS version 14.0 statistical software. 
Citation: Hsiao KC, Chao WR, Tsai JP, Wen MC, Lian JD, et al. (2014) Dysregulation of Interleukin 23 Receptor Expression in Kidney Allografts Associated with Composite Outcome. J Cytol Histol S4: 017. doi:10.4172/2157-7099.S4-017

Page 3 of 8

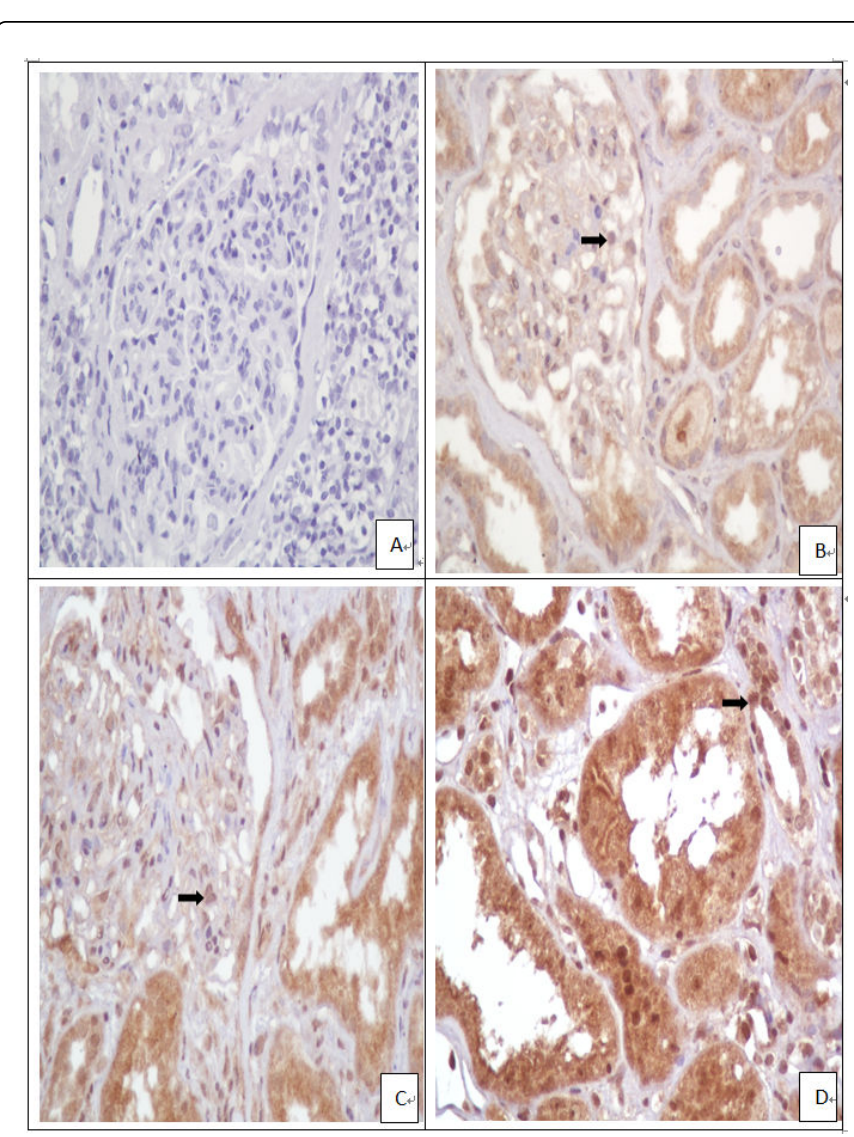

Figure 1: Representative panels showing different expression intensity of IHC IL-23R stain quantitative grading in glomeruli and renal tubules (IHC stain, $\times$ 400). (A) no IHC IL-23R stain in glomeruli and tubules, (B) mild IHC IL-23R stain in normal glomeurli and tubules, (C) moderate IHC IL-23R stain in atrophic glomeruli and tubules, and (D) high IHC IL-23R stain in normal renal tubules. The arrow indicates the IHC stain in a glomerular mesangial cell and renal tubular cell.

\section{Results}

We classified the 31 patients devided into group $A(n=15)$ and group $B(n=16)$. There were significant differences in the eGFR (ternimal), SCr (ternimal) and albumin level between those two groups. Our results indicate that group A had low ternimal eGFR (15.6 $\mathrm{mL} / \mathrm{min}$ for group A, $58.5 \mathrm{~mL} / \mathrm{min}$ for group $\mathrm{B}, \mathrm{p}<0.001$ ), high ternimal SCr $(3.3 \mathrm{mg} / \mathrm{dl}$ for group A, $1.2 \mathrm{mg} / \mathrm{dl}$ for group $\mathrm{B}, \mathrm{p}<0.001)$ and lower albumin levels $(3.5 \mathrm{~g} / \mathrm{dL}$ for group $\mathrm{A}, 3.8 \mathrm{~g} / \mathrm{dL}$ for group $\mathrm{B}$, $\mathrm{p}=0.025$ ) (Table 1).

\begin{tabular}{|l|l|l|l|l|l|}
\hline & \multicolumn{2}{|l|}{$\begin{array}{l}\text { Patients } \\
\text { reaching } \\
\text { composite } \\
\text { outcome } \\
\text { (group A) }\end{array}$} & \multicolumn{2}{l|}{$\begin{array}{l}\text { Patients } \\
\text { reaching } \\
\text { composite } \\
\text { outcome } \\
\text { (group B) }\end{array}$} & P value \\
\hline & median & $\begin{array}{l}25 \%-7 \\
5 \%\end{array}$ & median & $\begin{array}{l}25 \%-75 \\
\%\end{array}$ & \\
\hline Patient number $(\mathrm{n})$ & 15 & & 16 & & \\
\hline
\end{tabular}

\begin{tabular}{|c|c|c|c|c|c|}
\hline Age (year) & 57.3 & $\begin{array}{l}52.6 \\
60\end{array}$ & 55.85 & $\begin{array}{l}48.8- \\
62.23\end{array}$ & 0.861 \\
\hline Gender F/M (n) & $5 / 10$ & & $5 / 11$ & & 1 \\
\hline HTN (n, \%) & $12(80)$ & & $11(68.8)$ & & 0.685 \\
\hline $\mathrm{DM}(\mathrm{n}, \%)$ & $5(33.3)$ & & $7(43.8)$ & & 0.822 \\
\hline $\operatorname{HBV}(\mathrm{n}, \%)$ & $1(6.7)$ & & $2(12.5)$ & & 1 \\
\hline $\operatorname{HCV}(n, \%)$ & $6(40)$ & & $2(12.5)$ & & 0.113 \\
\hline Smoke (n, \%) & $6(40)$ & & $7(43.8)$ & & 0.833 \\
\hline BMI $\left(\mathrm{kg} / \mathrm{m}^{2}\right)$ & 27 & $\begin{array}{c}20.8- \\
30.6\end{array}$ & 24.9 & $\begin{array}{l}22.75- \\
26.78\end{array}$ & 0.338 \\
\hline $\mathrm{HD} / \mathrm{PD}(\mathrm{n})$ & $12 / 3$ & & $13 / 3$ & & 1 \\
\hline Dialysis duration (y) & 1 & $\begin{array}{l}0.7 \\
2.0\end{array}$ & 2 & $\begin{array}{c}0.93- \\
4.38\end{array}$ & 0.216 \\
\hline Pathologic diagnosis & & & & & 0.302 \\
\hline $\begin{array}{l}\text { (1) Acute tubular injury (n, } \\
\%)\end{array}$ & $1(6.7)$ & & $4(25)$ & & \\
\hline $\begin{array}{l}\text { (2) Mild cellular rejection } \\
(\mathrm{n}, \%)\end{array}$ & $4(26.7)$ & & $4(25)$ & & \\
\hline 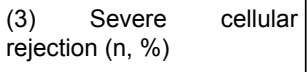 & $6(40)$ & & $7(43.8)$ & & \\
\hline $\begin{array}{l}\text { (4) Antibody rejection (n, } \\
\%)\end{array}$ & $4(26.7)$ & & $1(6.3)$ & & \\
\hline $\begin{array}{l}\text { Drug } \\
\text { Cyclosporin) }\end{array}$ & $14 / 1$ & & $13 / 3$ & & 0.6 \\
\hline $\mathrm{SBP}(\mathrm{mmHg})$ & 129 & $\begin{array}{l}120 \\
144\end{array}$ & 132 & $\begin{array}{l}120.5- \\
150\end{array}$ & 0.379 \\
\hline $\mathrm{DBP}(\mathrm{mmHg})$ & 74 & $60-82$ & 81 & $\begin{array}{l}70.75 \\
93\end{array}$ & 0.11 \\
\hline eGFR (biopsy) (ml/min) & 31 & $24-39$ & 34.5 & $\begin{array}{l}31.25- \\
44.75\end{array}$ & 0.202 \\
\hline eGFR (baseline) (ml/min) & 53 & $41-58$ & 60 & ${ }^{51} 67.75-$ & 0.093 \\
\hline eGFR (terminal) (ml/min) & 15.6 & $\begin{array}{l}8.6 \\
36.1\end{array}$ & 58.5 & $\begin{array}{l}44.75- \\
70.58\end{array}$ & $<0.001^{*}$ \\
\hline $\mathrm{SCr}$ (biopsy) (mg/dl) & 2.2 & $1.8-2.8$ & 1.8 & $\begin{array}{l}1.7 \\
2.28\end{array}$ & 0.216 \\
\hline $\mathrm{SCr}$ (baseline) (mg/dl) & 1.4 & $1.3-1.8$ & 1.2 & $\begin{array}{c}1.1 \\
1.4\end{array}$ & 0.066 \\
\hline $\mathrm{SCr}$ (terminal) (mg/dl) & 3.3 & $2.0-6.8$ & 1.2 & $\begin{array}{c}1.03- \\
1.6\end{array}$ & $<0.001^{*}$ \\
\hline Hemoglobin (g/dl) & 10.8 & $9.6-13$ & 11.65 & $\begin{array}{l}8.88 \\
13.43\end{array}-$ & 0.892 \\
\hline HbA1C (\%) & 6.4 & $\begin{array}{l}6.08 \\
7.08\end{array}$ & 6.3 & $\begin{array}{c}5.9 \\
7.9\end{array}$ & 0.914 \\
\hline $\begin{array}{l}\text { Low-density lipoprotein } \\
(\mathrm{mg} / \mathrm{dl})\end{array}$ & 86.5 & $\begin{array}{c}74.3- \\
121.5\end{array}$ & 84 & $\begin{array}{l}51 \\
132\end{array}$ & 0.683 \\
\hline Total cholesterol (mg/dl) & 161 & $\begin{array}{c}132 \\
187\end{array}$ & 179.5 & $\begin{array}{c}112.5- \\
243.25\end{array}$ & 0.682 \\
\hline
\end{tabular}


Citation: Hsiao KC, Chao WR, Tsai JP, Wen MC, Lian JD, et al. (2014) Dysregulation of Interleukin 23 Receptor Expression in Kidney Allografts Associated with Composite Outcome. J Cytol Histol S4: 017. doi:10.4172/2157-7099.S4-017

Page 4 of 8

\begin{tabular}{|c|c|c|c|c|c|}
\hline Triglyceride (mg/dl) & 112 & $93-181$ & 138.5 & $\begin{array}{c}113.5- \\
269.25\end{array}$ & 0.318 \\
\hline Albumin (mg/dl) & 3.5 & $\begin{array}{c}3.15 \\
3.75\end{array}$ & 3.8 & $\begin{array}{c}3.6 \\
4.1\end{array}$ & $0.025^{*}$ \\
\hline Uric acid (mg/dl) & 7.1 & $5.8-8.3$ & 6.5 & $\begin{array}{ll}5.7 \\
7.68\end{array}$ & 0.65 \\
\hline $\begin{array}{l}\text { Urine dipstick protein } \\
(\mathrm{mg} / \mathrm{dL})\end{array}$ & & & & & 0.296 \\
\hline $0 \mathrm{mg} / \mathrm{dL}$ & & $5(33.3)$ & $\begin{array}{l}10 \\
(62.5)\end{array}$ & & \\
\hline $30-100 \mathrm{mg} / \mathrm{dL}$ & & $7(46.7)$ & $\begin{array}{l}5 \\
(31.25)\end{array}$ & & \\
\hline$>100 \mathrm{mg} / \mathrm{dL}$ & & $3(20)$ & $1(6.25)$ & & \\
\hline \multicolumn{6}{|c|}{$\begin{array}{l}\text { Composite outcome: first event with doubling of } \mathrm{SCr} \text { or declining eGFR more } \\
\text { than } 30 \% \text {. HTN, hypertension; DM, diabetes mellitus; HBV, hepatitis B; HCV, } \\
\text { hepatitis C; BMI, body mass index; HD, hemodialysis; PD, peritoneal dialysis; } \\
\text { SBP, systolic blood pressure; DBP, diastolic blood pressure; eGFR, estimated } \\
\text { glomerular filtration; SCr: serum creatinine; HbA1C: hemoglobin A1c. }\end{array}$} \\
\hline *: $p<0.05$ indicates signific & & & & & \\
\hline
\end{tabular}

Table 1: Demographic and clinical characteristics of patients divided by patients reaching composite outcome (left) and patients not reaching composite outcome (right).

We compared the association between group A and group B with the intensity of IL-23/IL-23R expression in each component of the specimen in Table 2. However, there was no relationship found for thedifferent regions of renal tissues including the glomerular, tubular and intertisium.

\begin{tabular}{|l|l|l|l|l|}
\hline & Score & $\begin{array}{l}\text { Patients } \\
\text { reaching } \\
\text { composite } \\
\text { outcome } \\
\text { (group A) }\end{array}$ & $\begin{array}{l}\text { Patients not } \\
\text { reaching } \\
\text { composite } \\
\text { outcome } \\
\text { (group B) }\end{array}$ & P value \\
\hline IFS & Without & $5(33.3)$ & $9(60)$ & 0.143 \\
\hline & With & $10(66.7)$ & $6(40)$ & \\
\hline GFS & Without & $12(80)$ & $15(100)$ & 0.224 \\
\hline IL23 (Gn)n & With & $3(20)$ & 0 & \\
\hline Without & $6(54.5)$ & $9(81.8)$ & 0.361 \\
\hline IL23 (Gn)c & With & $5(45.5)$ & $2(18.2)$ & \\
\hline & Without & $9(81.8)$ & $5(45.5)$ & 0.183 \\
\hline IL23 (Ga)n & Without & $2(18.2)$ & $6(54.5)$ & \\
\hline & With & $1(7.7)$ & 0 & 1 \\
\hline IL23 (Ga)c & Without & $10(76.9)$ & $6(100)$ & 0.517 \\
\hline & With & $3(23.1)$ & 0 & \\
\hline IL23 (ATn)n & Without & $15(100)$ & $15(93.8)$ & 1 \\
\hline
\end{tabular}

\begin{tabular}{|c|c|c|c|c|}
\hline & With & 0 & $1(6.3)$ & \\
\hline \multirow[t]{2}{*}{ IL23 (ATn)c } & Without & 0 & $1(6.3)$ & 1 \\
\hline & With & $15(100)$ & $15(93.8)$ & \\
\hline \multirow[t]{2}{*}{ IL23 (ATa)C } & Without & 0 & $1(33.3)$ & 0.3 \\
\hline & With & $7(100)$ & $2(66.7)$ & \\
\hline \multirow[t]{2}{*}{ IL23 (IT)c } & Without & $14(93.3)$ & $11(68.8)$ & 0.172 \\
\hline & With & $1(6.7)$ & $5(31.3)$ & \\
\hline \multirow[t]{2}{*}{ IL23R (Gn)n } & Without & $4(44.4)$ & $3(30)$ & 0.65 \\
\hline & With & $5(55.6)$ & $7(70)$ & \\
\hline \multirow[t]{2}{*}{ IL23R (Gn)c } & Without & $8(88.9)$ & $8(80)$ & 1 \\
\hline & With & $1(11.1)$ & $2(20)$ & \\
\hline \multirow[t]{2}{*}{ IL23R (Ga)n } & Without & $9(69.2)$ & $5(71.4)$ & 1 \\
\hline & With & $4(30.8)$ & $2(28.6)$ & \\
\hline \multirow[t]{2}{*}{ IL23R (Ga)c } & Without & $13(100)$ & $5(71.4)$ & 0.111 \\
\hline & With & 0 & $2(28.6)$ & \\
\hline \multirow[t]{2}{*}{ IL23R (ATn)n } & Without & $9(60)$ & $8(50)$ & 0.576 \\
\hline & With & $6(40)$ & $8(50)$ & \\
\hline \multirow[t]{2}{*}{ IL23R (ATn)C } & Without & $2(13.3)$ & $3(18.8)$ & 1 \\
\hline & With & $13(86.7)$ & $13(81.3)$ & \\
\hline \multirow[t]{2}{*}{ IL23R (ATa)n } & Without & $3(50)$ & $3(75)$ & 0.571 \\
\hline & With & $3(50)$ & $1(25)$ & \\
\hline \multirow[t]{2}{*}{ IL23R (ATa)C } & Without & $1(16.7)$ & $1(25)$ & 1 \\
\hline & With & $5(83.3)$ & $3(75)$ & \\
\hline \multirow[t]{2}{*}{ IL23R (IT)n } & Without & $10(66.7)$ & $13(81.3)$ & 0.433 \\
\hline & With & $5(33.3)$ & $3(18.8)$ & \\
\hline \multirow[t]{2}{*}{ IL23R (IT)C } & Without & $10(66.7)$ & $9(56.3)$ & 0.552 \\
\hline & With & $5(33.3)$ & $7(43.8)$ & \\
\hline \multicolumn{5}{|c|}{$\begin{array}{l}\text { Composite outcome: first event with doubling of } \mathrm{SCr} \text { or declining eGFR more } \\
\text { than } 30 \% \text { IFS, interstitial fibrosis score; GFS, glomerular fibrosis score; (Gn)n, } \\
\text { nuclear staining intensity of normal glomerulus mesangial cell; (Gn)c, } \\
\text { cytoplasmic staining intensity of normal glomerulus mesangial cell; (Ga)n, } \\
\text { nuclear staining intensity of atrophy glomerulus mesangial cell; (Ga)c, } \\
\text { cytoplasmic staining intensity of atrophy glomerulus mesangial cell; (ATn)n, } \\
\text { nuclear staining intensity of normal renal tubule; (ATn)c, cytoplasmic staining } \\
\text { intensity of normal renal tubule; (ATa)n, nuclear staining intensity of atrophy } \\
\text { renal tubule; (ATa)c, cytoplasmic staining intensity of atrophy renal tubule; (IT)n, } \\
\text { nuclear staining intensity of interstitium; (IT)c, cytoplasmic staining intensity of } \\
\text { interstitium. }\end{array}$} \\
\hline & & & & \\
\hline
\end{tabular}

Table 2: Intensity of IL 23/IL-23 receptor expression in different regions of renal tissues divided by patient reach composite outcome (left) and patient did not reach composite outcome (right).

The Spearman's correlation between the clinical variables and primary composite outcome revealed that group A was negatively 
Citation: Hsiao KC, Chao WR, Tsai JP, Wen MC, Lian JD, et al. (2014) Dysregulation of Interleukin 23 Receptor Expression in Kidney Allografts Associated with Composite Outcome. J Cytol Histol S4: 017. doi:10.4172/2157-7099.S4-017

Page 5 of 8

associated with eGFR (ternimal) $(\mathrm{r}=0.78, \mathrm{p}<0.001)$, positively associated with $\mathrm{SCr}$ (terminal) $(\mathrm{r}=0.784, \mathrm{p}<0.001)$, and negatively associated with albumin level ( $\mathrm{r}=0.427 ; \mathrm{p}=0.024)$ (Table 3$)$.

\begin{tabular}{|c|c|c|}
\hline & $\mathbf{R}$ & $P$ value \\
\hline Patient number (n) & 0.032 & 0.862 \\
\hline Age (year) & -0.222 & 0.905 \\
\hline Gender F/M (n) & 0.128 & 0.491 \\
\hline HTN (n, \%) & 0.081 & 0.666 \\
\hline $\mathrm{DM}(\mathrm{n}, \%)$ & -0.099 & 0.598 \\
\hline $\operatorname{HBV}(n, \%)$ & 0.314 & 0.085 \\
\hline $\operatorname{HCV}(n, \%)$ & -0.38 & 0.839 \\
\hline Smoke $(n, \%)$ & 0.177 & 0.341 \\
\hline BMI (kg/m2) & 0.016 & 0.933 \\
\hline HD/PD (n) & -0.23 & 0.213 \\
\hline Pathology diagnosis & 0.281 & 0.125 \\
\hline Drug & 0.18 & 0.332 \\
\hline $\mathrm{SBP}(\mathrm{mmHg})$ & -0.166 & 0.371 \\
\hline DBP (mmHg) & -0.296 & 0.106 \\
\hline eGFR (biopsy) (ml/min) & -0.238 & 0.196 \\
\hline eGFR (baseline) (ml/min) & -0.307 & 0.093 \\
\hline eGRF (terminal) (ml/min) & -0.78 & $<0.001^{*}$ \\
\hline SCr (biopsy) (mg/dl) & 0.232 & 0.21 \\
\hline SCr (baseline) (mg/dl) & 0.342 & 0.06 \\
\hline $\mathrm{SCr}$ (terminal) (mg/dl) & 0.784 & $<0.001^{*}$ \\
\hline Hemoglobin (g/dl) & 0.029 & 0.877 \\
\hline HbA1C (\%) & 0.025 & 0.898 \\
\hline Low-density lipoprotein (mg/dl) & 0.078 & 0.686 \\
\hline Total cholesterol (mg/dl) & -0.079 & 0.671 \\
\hline Triglyceride (mg/dl) & -0.184 & 0.322 \\
\hline Albumin (mg/dl) & -0.427 & 0.024 \\
\hline Uric acid (mg/dl) & 0.091 & 0.638 \\
\hline Urine dipstick protein (mg/dL) & 0.309 & 0.09 \\
\hline \multicolumn{3}{|c|}{$\begin{array}{l}\text { Composite outcome: first event with doubling of } \mathrm{SCr} \text { or declining eGFR more } \\
\text { than } 30 \% \text {. HTN, hypertension; DM, diabetes mellitus; HBV, hepatitis B; HCV, } \\
\text { hepatitis C; BMI, body mass index; HD, hemodialysis; PD, peritoneal dialysis; } \\
\text { SBP, systolic blood pressure; DBP, diastolic blood pressure; eGFR, estimated } \\
\text { glomerular filtration; SCr, serum creatinine; HbA1C, hemoglobin A1c. }\end{array}$} \\
\hline
\end{tabular}

Table 3: Spearman's correlation association between clinical variables when reaching composite outcome
Table 4 shows the Spearman's correlation between pathological variables and primary composite outcome. Univariate analysis indicated that IHC IL-23R expression intensity stain was negatively associated with atrophic glomerular mesangial cell cytoplasm $(r=0.454$, $\mathrm{p}=0.044)$.

\begin{tabular}{|l|l|l|}
\hline & $\mathbf{R}$ & P value \\
\hline IFS & 0.267 & 0.153 \\
\hline GFS & 0.333 & 0.072 \\
\hline IL23 (Gn)n & 0.293 & 0.186 \\
\hline IL23 (Gn)c & -0.378 & 0.083 \\
\hline IL23 (Ga)n & 0.16 & 0.513 \\
\hline IL23 (Ga)c & 0.294 & 0.222 \\
\hline IL23 (ATn)n & -0.177 & 0.341 \\
\hline IL23 (ATn)c & 0.177 & 0.341 \\
\hline IL23 (ATa)c & 0.509 & 0.133 \\
\hline IL23 (IT)c & -0.311 & 0.089 \\
\hline IL23R (Gn)n & -0.15 & 0.541 \\
\hline IL23R (Gn)c & -0.122 & 0.62 \\
\hline IL23R (Ga)n & 0.023 & 0.924 \\
\hline IL23R (Ga)c & -0.454 & $0.044^{*}$ \\
\hline IL23R (ATn)c & 0.074 & 0.694 \\
\hline IL23R (ATa)n & 0.25 & 0.486 \\
\hline IL23R (ATa)c & 0.102 & 0.779 \\
\hline IL23R (IT)n & 0.167 & 0.371 \\
\hline IL23R (IT)c & -0.107 & 0.567 \\
\hline COmpost & \\
\hline
\end{tabular}

Composite outcome: first event with doubling of SCr or declining eGFR more than $30 \%$. IFS, interstitial fibrosis score; GFS, glomerular fibrosis score; (Gn)n, nuclear staining intensity of normal glomerulus mesangial cell; $(G n) c$, cytoplasmic staining intensity of normal glomerulus mesangial cell; (Ga)n, nuclear staining intensity of atrophy glomerulus mesangial cell; (Ga)c, cytoplasmic staining intensity of atrophy glomerulus mesangial cell; (ATn)n, nuclear staining intensity of normal renal tubule; (ATn)c: cytoplasmic staining intensity of normal renal tubule; (ATa)n, nuclear staining intensity of atrophy renal tubule; (ATa)c, cytoplasmic staining intensity of atrophy renal tubule; (IT)n, nuclear staining intensity of interstitium; (IT)c,cytoplasmic staining intensity of interstitium.

*: $p<0.05$ indicates significance

Table 4: Spearman's correlation association about pathological variables when reaching composite outcome.

Figure 2 illustrates the different expression intensities of H\&E stain (panel A, C, E) and IL-23 IHC stain (panel B, D, F) in pateints reaching composite outcome; H\&E and IL-23R IHC stain in patients not reaching composite outcome is shown in Figure 3. Finally, a decreased intensity of IL-23R expression in atrophic glomerular mesangial cell cytoplasma in group A (panel A) can be seen in Figure 4 . 
Citation: Hsiao KC, Chao WR, Tsai JP, Wen MC, Lian JD, et al. (2014) Dysregulation of Interleukin 23 Receptor Expression in Kidney Allografts Associated with Composite Outcome. J Cytol Histol S4: 017. doi:10.4172/2157-7099.S4-017

Page 6 of 8

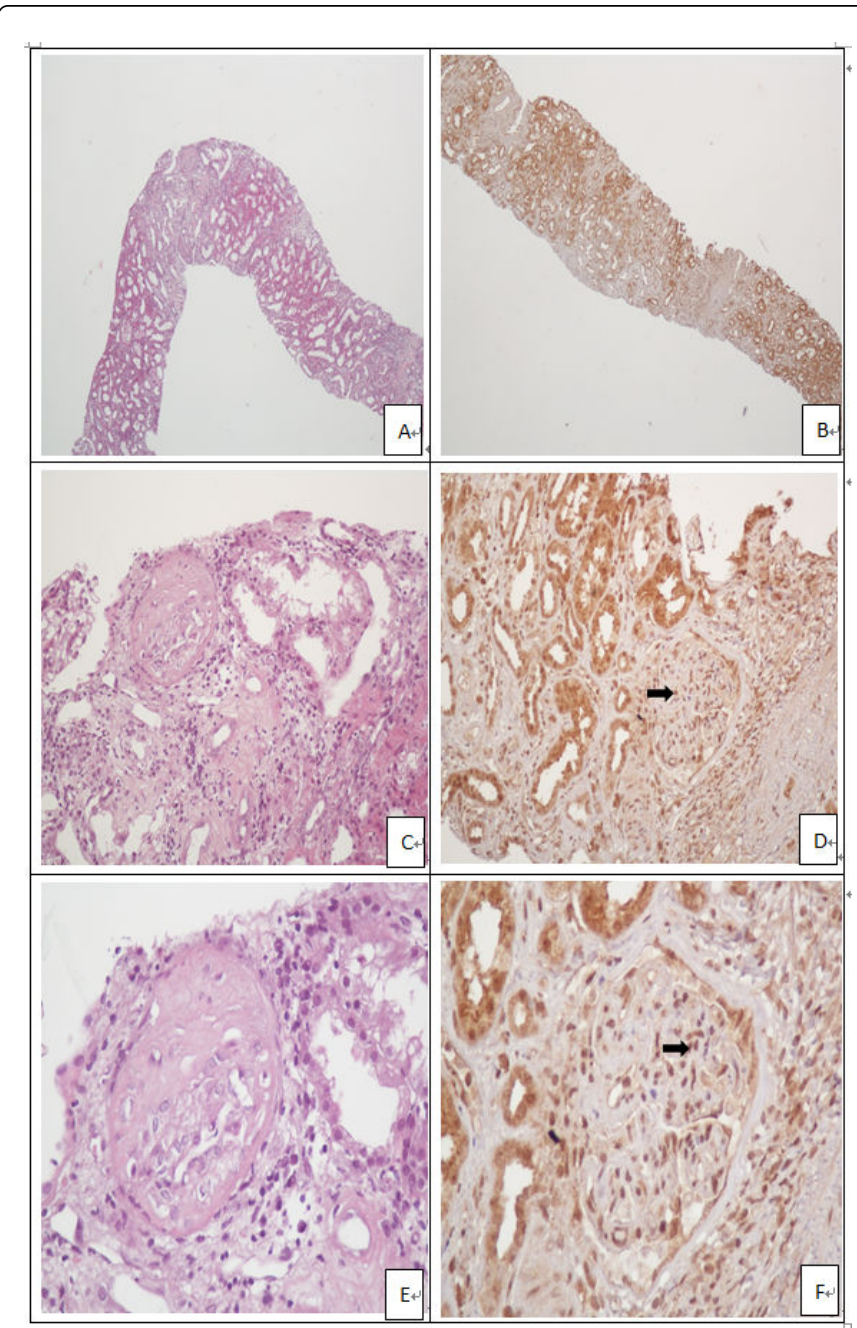

Figure 2: Representative panels showing a different intensity of IL-23 expression by IHC stain compared to H\&E stain in a patient reaching composite outcome (group A). (A) H\&E stain, $x$ 20, (B) IHC IL-23 stain, $x$ 20, (C) H\&E stain, $x$ 200, (D) IHC IL-23 stain, $x$ 200, (E) H\&E stain, $x$ 400, (F) IHC IL-23 stain, $x$ 400. The arrow indicates the IL-23 IHC stain in a glomerular mesangial cell.

\section{Discussion}

Our results demonstrate that patients reaching the composite outcome were negatively associated with atrophic glomerular mesangial cell cytoplasmic IL-23R expression $(\mathrm{p}=0.044)$. However, IL-23 expression was not associated with the outcome in any of the renal tissue, including the glomerular, tubule and interstitium.

In the clinical variable analysis, the group $\mathrm{A}$ was negatively associated with terminal eGFR $(r=0.78, \mathrm{p}<0.001)$, positively associated with terminal SCr $(r=0.784, p<0.001)$, and negatively associated with albumin level ( $r=0.427 ; p=0.024)$. Because the definition of composite outcome was doubling volume of SCr or a declining baseline eGFR of more than $30 \%$ in our study, the results of terminal Scr and eGFR could be due to the definition. Furthermore, compared with group B, group A had more ABMR $(26.7 \%$ vs. $6.3 \%)$ in the pathologial diagnosis and higher urine dipstick protein $(>100 \mathrm{mg} / \mathrm{dL}, 20 \%$ vs.
6.25\%). Given that AMBR usually presents with transplant glomerulopathy and proteinuria [22,23], the lower albumin level in group A $(3.5 \mathrm{mg} / \mathrm{dl}$ vs. $3.8 \mathrm{md} / \mathrm{dl}, \mathrm{p}=0.025)$ could be caused by a higher ABMR with transplant glomerulopathy and high urine dipstick protein in our study.

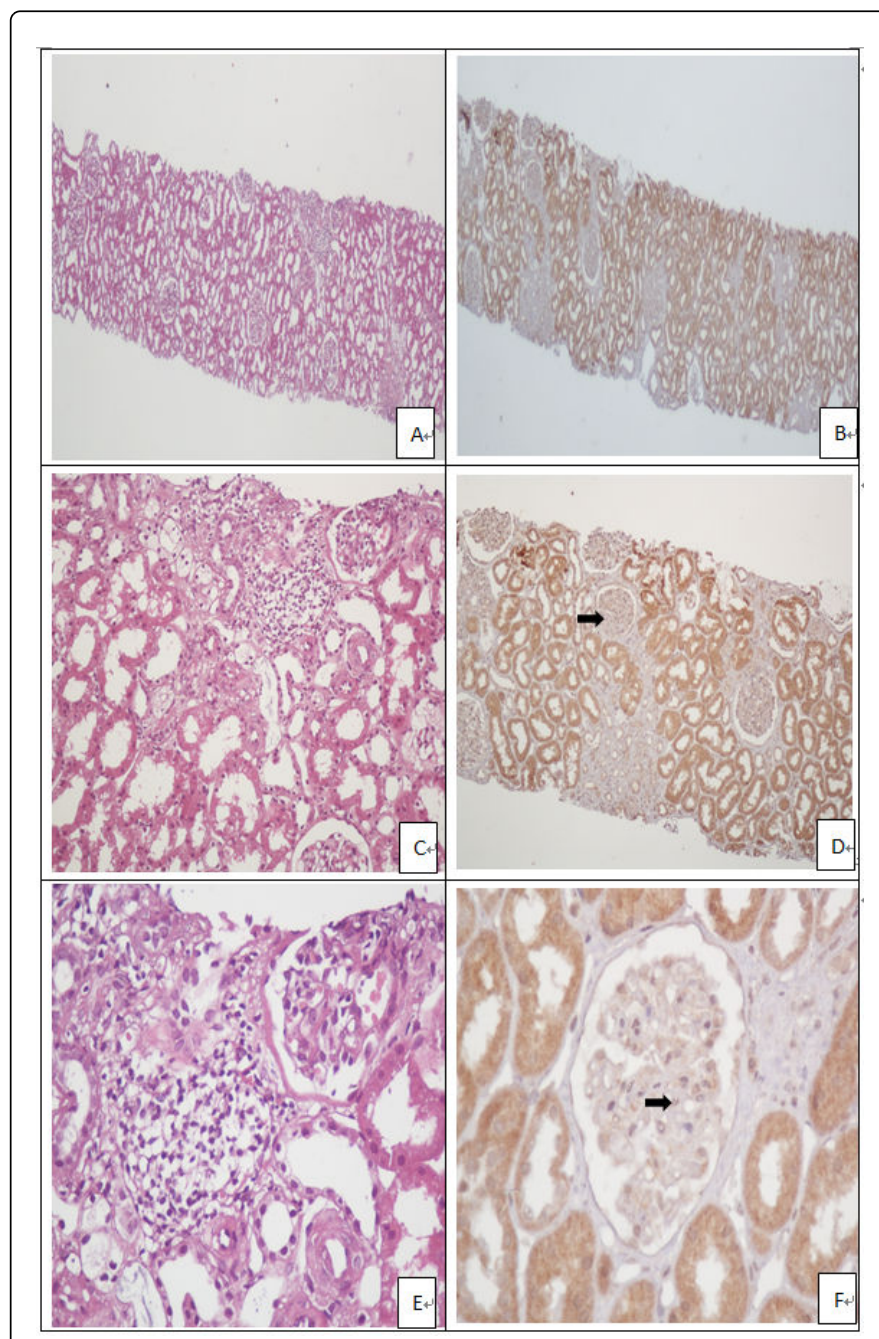

Figure 3: Representative panels showing a different intensity of IL-23R expression by IHC stain compared to the H\&E stain in a patien not reaching composite outcome (group B). (A) H\&E stain, x 20, (B) IHC IL-23R stain, x 20, (C) H\&E stain, x 200, (D) IHC IL-23R stain, $x$ 200, (E) H\&E stain, $x$ 400, (F) IHC IL-23R stain, $x$ 400. The arrow indicates the IL-23R IHC stain in a glomerular mesangial cell.

There have been several reports about interlukin marker expression in acute allograft rejection. Byung at al. revealed that higher infiltration by Th17 cells is associated with severe acute T-cellmediated graft rejection. Higher infiltration of Th17 is significantly associated with the severity of allograft dysfunction and tissue injury [24]. IL-17 expression by tubular epithelial cells in renal transplant recipients with acute antibody-mediated rejection has also been observed, with IL-17 tubular expression being directly and significantly correlated with the extension of C4d deposits [25]. 
Although interlukin expression was found to be approximally expressed in the renal tubule and interstitium in previous research, several studies have revealed the correction of IL-23 and glomeruli disease. Paust et al. reported that the IL-23/Th17 axis contributes to renal injury in experimental glomerulonephritis, with IL-17 enhancing the production of the proinflammatory chemokines CCL2/MCP-1, CCL3/MIP-1, and CCL20/LARC in mouse mesangial cells. They further found that IL-23 p19-/- mice developed less severe nephritis as measured by renal function, albuminuria, and frequency of glomerular crescent formation [26]. IL-23 receptor expression has also been shown to be up-regulated in lupus nephritis; the greater the increase in serum level of IL-23R, the greater the glomerular damage of lupus nephritis in mice [27].

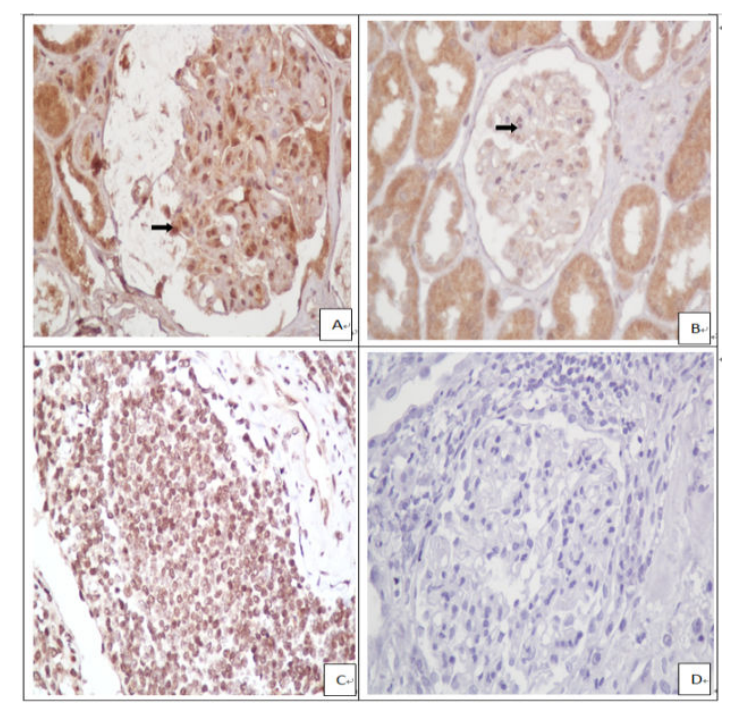

Figure 4: Representative panels showing a decreased intensity of IHC IL-23R expression in atrophic glomerular mesangial cell cytoplasma in a patient reaching composite outcome (group A) (IHC stain, x400). (A) IHC IL-23R stain in a patient reaching composite outcome (group A), (B) IHC IL-23R stain in a patient not reaching composite outcome (group B), (C) positive control of IL-23R IHC stain, (D) negative control of IL-23R IHC stain. The arrow indicates the IL-23R IHC stain in an atrophic glomerular mesangial cell.

In our study, the patients reaching the composite outcome had a decline of IL-23R expression in atrophic glomerular mesengial cell cytoplasm. ABMR was more evident with transplant glomerupathy [22]. Since our group A had a high ABMR ratio in the pathological diagnosis and greater deterioration of renal function, there could be more severe glomeruli demange with the presentation of atrophic glomeuli, and for this reason, less IL-23R was expressed in the atrophic glomerular mesangial cell cytoplasma. In other words, the more the allograft glomerular damage, the less the IL-23R expression in atrophic glomerular mesangial cell cytoplasm.

The limitation of this study was the small patient number, which caused inconsistency of the $p$ values for the IL-23R expression in the glomerular cytoplasma of atrophic mesangial cells as seen between Table 2 ( $\mathrm{p}=0.111$, by Fisher's exact test) and Table $4(\mathrm{p}=0.044$, by Spearman's correlation). To increase the patient number could further clarify the role of interleukin 23 receptor expression in kidney allografts.

In summary, our analysis of the expression of IL-23R in allograft specimens indicates a possible role of IL-23R over mesangial cytoplasm of atrophic glomerular cells. However, because most of the pathologic changes of acute allograft rejection were involved in the renal interstitium, the finding of IL-23R expression over glomeruli needs further study for clarification.

\section{References}

1. Meier-Kriesche HU, Schold JD, Srinivas TR, Kaplan B (2004) Lack of improvement in renal allograft survival despite a marked decrease in acute rejection rates over the most recent era. Am J Transplant 4: 378-383.

2. Trpkov K, Campbell P, Pazderka F, Cockfield S, Solez K, et al. (1996) Pathologic features of acute renal allograft rejection associated with donor-specific antibody, Analysis using the Banff grading schema. Transplantation 61: 1586-1592.

3. Afzali B, Lombardi G, Lechler RI, Lord GM (2007) The role of T helper 17 (Th17) and regulatory T cells (Treg) in human organ transplantation and autoimmune disease. Clin Exp Immunol 148: 32-46.

4. Colvin RB, Cohen AH, Saiontz C, Bonsib S, Buick M, et al. (1997) Evaluation of pathologic criteria for acute renal allograft rejection: reproducibility, sensitivity, and clinical correlation. Journal of the American Society of Nephrology 8: 1930-1941.

5. Akl A, Luo S, Wood KJ (2005) Induction of transplantation tolerance-the potential of regulatory T cells. Transpl Immunol 14: 225-230.

6. Mauiyyedi S, Crespo M, Collins AB, Schneeberger EE, Pascual MA, et al. (2002) Acute humoral rejection in kidney transplantation: II. Morphology, immunopathology, and pathologic classification. J Am Soc Nephrol 13: 779-787.

7. Feucht HE, Schneeberger H, Hillebrand G, Burkhardt K, Weiss M, et al. (1993) Capillary deposition of C4d complement fragment and early renal graft loss. Kidney international 43: 1333-1338.

8. Collins AB, Schneeberger EE, Pascual MA, Saidman SL, Williams WW, et al. (1999) Complement activation in acute humoral renal allograft rejection: diagnostic significance of $\mathrm{C} 4 \mathrm{~d}$ deposits in peritubular capillaries. J Am Soc Nephrol 10: 2208-2214.

9. Mosmann TR, Cherwinski H, Bond MW, Giedlin MA, Coffman RL (2005) Two types of murine helper T cell clone. I. Definition according to profiles of lymphokine activities and secreted proteins. Journal of immunology 175: 5-14.

10. Trinchieri G, Pflanz S, Kastelein RA (2003) The IL-12 family of heterodimeric cytokines: new players in the regulation of $\mathrm{T}$ cell responses. Immunity 19: 641-644.

11. Markey KA, MacDonald KP, Hill GR (2008) Impact of cytokine gene polymorphisms on graft-vs-host disease. Tissue Antigens 72: 507-516.

12. Chu CQ, Wittmer S, Dalton DK (2000) Failure to suppress the expansion of the activated CD4 $\mathrm{T}$ cell population in interferon gamma-deficient mice leads to exacerbation of experimental autoimmune encephalomyelitis. J Exp Med 192: 123-128.

13. Elmaagacli AH, Koldehoff M, Landt O, Beelen DW (2008) Relation of an interleukin-23 receptor gene polymorphism to graft-versus-host disease after hematopoietic-cell transplantation. Bone Marrow Transplant 41: 821-826.

14. Duerr RH, Taylor KD, Brant SR, Rioux JD, Silverberg MS, et al. (2006) A genome-wide association study identifies IL23R as an inflammatory bowel disease gene. Science 314: 1461-1463.

15. Huber AK, Jacobson EM, Jazdzewski K, Concepcion ES, Tomer Y (2008) Interleukin (IL)-23 receptor is amajor susceptibility gene for Graves' ophthalmopathy: the IL-23/T-helper 17 axis extends to thyroid autoimmunity. J Clin Endocrinol Metab 93: 1077-1081. 
Citation: Hsiao KC, Chao WR, Tsai JP, Wen MC, Lian JD, et al. (2014) Dysregulation of Interleukin 23 Receptor Expression in Kidney Allografts Associated with Composite Outcome. J Cytol Histol S4: 017. doi:10.4172/2157-7099.S4-017

Page 8 of 8

16. Faragó B, Magyari L, Sáfrány E, Csöngei V, Járomi L, et al. (2008) Functional variants of interleukin-23 receptor gene confer risk for rheumatoid arthritis but not for systemic sclerosis. Ann Rheum Dis 67: 248-250.

17. Tsai JP, Yang SF, Wu SW, Hung TW, Tsai HC, et al. (2011) Association between interleukin 23 receptor polymorphism and kidney transplant outcomes: a 10-year Taiwan cohort study. Clin Chim Acta 412: 958-962.

18. National Kidney Foundation (2002) K/DOQI clinical practice guidelines for chronic kidney disease: evaluation, classification, and stratification. Am J Kidney Dis 39: S1-266.

19. Knoll GA, Cantarovitch M, Cole E, Gill J, Gourishankar S, et al. (2008) The Canadian ACE-inhibitor trial to improve renal outcomes and patient survival in kidney transplantation-study design. Nephrology Dialysis Transplantation 23: 354-358.

20. Fellström B, Holdaas H, Jardine AG, Nyberg G, Grönhagen-Riska C, et al. (2005) Risk factors for reaching renal endpoints in the assessment of Lescol in renal transplantation (ALERT) trial. Transplantation 79: 205-212.

21. National Kidney Foundation (2014) GFR decline as an endpoint in clinical trials for CKD.
22. Haas M, Sis B, Racusen LC, Solez K, Glotz D, et al. (2014) Banff 2013 meeting report: inclusion of c4d-negative antibody-mediated rejection and antibody-associated arterial lesions. Am J Transplant 14: 272-283.

23. Djamali A, Kaufman DB, Ellis TM, Zhong W, Matas A, et al. (2014) Diagnosis and management of antibody-mediated rejection: current status and novel approaches. Am J Transplant 14: 255-271.

24. Chung BH, Oh HJ, Piao SG, Sun IO, Kang SH, et al. (2011) Higher infiltration by Th17 cells compared with regulatory $\mathrm{T}$ cells is associated with severe acute T-cell-mediated graft rejection. Exp Mol Med 43: 630-637.

25. Loverre A, Tataranni T, Castellano G, Divella C, Battaglia M, et al. (2011) IL-17 expression by tubular epithelial cells in renal transplant recipients with acute antibody-mediated rejection. Am J Transplant 11: 1248-1259.

26. Paust HJ, Turner JE, Steinmetz OM, Peters A, Heymann F, et al. (2009) The IL-23/Th17 axis contributes to renal injury in experimental glomerulonephritis. J Am Soc Nephrol 20: 969-979.

27. Zhang Z, Kyttaris VC, Tsokos GC (2009) The role of IL-23/IL-17 axis in lupus nephritis. J Immunol 183: 3160-3169. 